\title{
Impulsive prion disease model
}

\author{
Abdelkader Lakmeche ${ }^{1, \mathrm{a}}$, Mohamed Helal ${ }^{1, \mathrm{~b}}$, Imane Mammar ${ }^{1, \mathrm{c}}$, and Abdelghani Ouahab ${ }^{2, \mathrm{~d}}$ \\ ${ }^{1}$ Laboratory of Biomathematics, Univ. Sidi Bel Abbes, PB. 89, Sidi Bel Abbes 22000, Algeria \\ ${ }^{2}$ Laboratory of Mathematics, Univ. Sidi Bel Abbes, PB. 89, Sidi Bel Abbes 22000, Algeria
}

\begin{abstract}
A model of prion diseases with impulse effects is studied in this work. First we transform the model to a system of three differential equations with impulse effects in order to study the stability of periodic solution. After that we study the general model by the mean of evolution semi group in order to find conditions of existence of mild solution.
\end{abstract}

\section{Introduction}

In this work we consider a model describing prion polymerization, our model is inspired from those of Webb and collaborators (see [3], [6]), it is constituted by a differential equation modeling the evolution of $\operatorname{Pr} P^{c}$ and a partial differential equation describing the $\operatorname{Pr} P^{s c}$ evolution. We consider the case where the monomers are produced discretely at fixed times $t_{i}$, which is expressed by impulse equations (see [4]).

More specifically we consider the following system

$$
\left\{\begin{array}{l}
v^{\prime}(t)=-\gamma v(t)-\tau v(t) \int_{x_{0}}^{\infty} u(t, x) d x \\
\quad+2 \int_{0}^{x_{0}} x \int_{x_{0}}^{\infty} \beta(y) \kappa(x, y) u(t, y) d y d x, t \neq t_{i}, \quad i=1,2, \ldots, \\
v\left(t_{i}^{+}\right)-v\left(t_{i}^{-}\right)=\lambda_{i}, \quad \lambda_{i}>0, \quad i=1,2, \ldots, \\
\partial_{t} u(t, x)+\tau v(t) \partial_{x} u(t, x)+(\mu(x)+\beta(x)) u(t, x) \\
\quad=2 \int_{x}^{\infty} \beta(y) \kappa(x, y) u(t, y) d y
\end{array}\right.
$$

for $t \geq 0, x \in\left[x_{0},+\infty\right)$ with fixed $x_{0}>0$.

\section{The variables and parameters of the model are}

- $v(t)$ is the number of $\operatorname{Pr} P^{c}$ monomers at time t,

- $u(t, x)$ is the density of $\operatorname{Pr} P^{s c}$ polymers of length $x$ at time $t$,

\footnotetext{
a e-mail: lakmeche@yahoo.fr

b e-mail: mhelal_abbes@yahoo.fr

c e-mail: i.mammar@yahoo.fr

de-mail: agh_ouahab@yahoo.fr
}

This is an Open Access article distributed under the terms of the Creative Commons Attribution License 4.0, which permits unrestricted use, distribution, and reproduction in any medium, provided the original work is properly cited. 
- $x_{0}$ is the lower bound for polymer length (that is polymers have length $x$ with $x_{0}<x<\infty$ ),

- $\lambda_{i}$ is the number of monomers $\operatorname{Pr} P^{c}$ produced at time $t_{i}$,

- $\gamma$ is the metabolic degradation rate for $\operatorname{Pr} P^{c}$,

- $\tau$ is the rate associated with lengthening of $\operatorname{Pr} P^{s c}$ polymers by attaching to and converting $\operatorname{Pr} P^{c}$ monomers,

- $\beta(x)$ is length-dependent rate of polymer breakage,

- $\kappa(x, y)$ is the probability, when a polymer of length $y$ breaks, that one of the two resulting polymers has length $x$,

- $\mu(x)$ is the length-dependent metabolic degradation rate of $\operatorname{Pr} P^{s c}$ polymers having length $x$.

The kernel $\kappa(y, x)$ should satisfy the following properties

$$
\kappa(y, x) \geq 0, \kappa(y, x)=\kappa(x-y, x), \quad \int_{0}^{x} \kappa(y, x) d y=1,
$$

for all $x \geq x_{0}, y \geq 0$,

$$
\begin{array}{ll}
\kappa(y, x)=1 / x, & \text { if } x>x_{0} \text { and } 0<y<x . \\
\kappa(y, x)=0, & \text { elsewhere. }
\end{array}
$$

In the following section we transform the model (1) to a system of impulsive differential equations in order to study the stability of periodic trivial solutions, in the third section we study the general model. In the last section we give some remarks.

\section{Conversion to impulsive differential equations}

Define the functions $V(t)=v(t), U(t)=\int_{x_{0}}^{\infty} u(t, y) d y$ and $P(t)=\int_{x_{0}}^{\infty} y u(t, y) d y$ where $U(t)$ is the total number of polymers and $P(t)$ is the total number of monomers in polymers at time $t$.

We deduce from model (1) the following system of impulsive differential equations

$$
\begin{aligned}
\dot{V}(t) & =\lambda-\gamma V(t)-\tau V(t) U(t)+2 \beta x_{0}^{2} U(t)=F_{1}(V(t), U(t), P(t)), \\
\dot{U}(t) & =-\mu U(t)+\beta P(t)-2 \beta x_{0} U(t)=F_{2}(V(t), U(t), P(t)) \\
\dot{P}(t) & =\tau V(t) U(t)-\mu P(t)-\beta x_{0}^{2} U(t)=F_{3}(V(t), U(t), P(t)) \\
V\left(t_{i}^{+}\right) & =V\left(t_{i}\right)+\lambda_{i}=\Theta_{1}\left(V\left(t_{i}\right), U\left(t_{i}\right), P\left(t_{i}\right)\right), \lambda_{i}>0, i \in \mathbb{N}^{*} \\
U\left(t_{i}^{+}\right) & =U(t)=\Theta_{2}\left(V\left(t_{i}\right), U\left(t_{i}\right), P\left(t_{i}\right)\right) \\
P\left(t_{i}^{+}\right) & =P(t)=\Theta_{3}\left(V\left(t_{i}\right), U\left(t_{i}\right), P\left(t_{i}\right)\right) .
\end{aligned}
$$

In this section we consider the case where $\lambda_{i}=\lambda_{0}>0$ and $t_{i}=\tau \forall i \in \mathbb{N}^{*}$.

We are interested by the existence and stability of periodic solutions of (2).

A solution $\xi=(V, U, P)$ of the problem (2) is a function defined in $\mathbb{R}_{+}$, with non-negative components, continuously differentiable in $\mathbb{R}_{+}-\left\{t_{i}\right\}_{i \geq 0}$, with $t_{0}=0$ and satisfying (2).

The function $\xi=(V, 0,0)$ is the trivial solution of problem (2) where $V(t)=V_{0} e^{-\gamma t}$. Also $\xi$ is called trivial $T_{0}$-periodic solution if it is a trivial solution with $\xi\left(n T_{0}\right)=\xi\left((n+1) T_{0}\right)$ for all $n \geq 0$.

We have $F_{2}(V, 0,0) \equiv \Theta_{2}(V, 0,0) \equiv 0$ and $F_{2}(V, 0,0) \equiv \Theta_{2}(V, 0,0) \equiv 0$.

Our main objective is to study the stability of the trivial periodic solution and the loss of stability for some values of the parameters. 
Let $\Phi$ be the flow associated to (2), we have $\Phi\left(t, X_{0}\right)=\xi(t), 0<t \leq T_{0}$ where $\xi(0)=X_{0}=\xi(0)=$ $(V(0), U(0), P(0))$.

Let $\Psi$ be the operator defined by

$$
\Psi\left(\tau, X_{0}\right)=\Theta\left(\Phi\left(\tau, X_{0}\right)\right),
$$

we denote by $D_{X} \Psi$ the derivative of $\Psi$ with respect to $X$. Then $\xi=\Phi\left(\cdot, X_{0}\right)$ is a $\tau$-periodic solution of (2) if and only if $\Psi\left(\tau, X_{0}\right)=X_{0}$ i.e. $X_{0}$ is a fixed point of $\Psi(\tau, \cdot)$, and it is exponentially stable if $\rho\left(D_{X} \Psi(\tau, \cdot)\right)<1$.

Let $V_{s}$ be a $T_{0}$-periodic function defined by $V_{s}(t)=\lambda_{0} \frac{e^{-\gamma t}}{1-e^{-\gamma T_{0}}}$ for $0<t \leq T_{0}$.

For $U=P=0$ the problem (2) has $T_{0}$-periodic solution $\xi=\left(V_{s}, 0,0\right)$.

Consider the following hypothesis

(H1) $\max \left(\frac{\left(1-e^{-\gamma T_{0}}\right)}{\beta \tau}\left[2 \beta^{2} x_{0}^{2}-\left(\frac{2}{T_{0}}-\left(\mu+\beta x_{0}\right)\right)^{2}\right], \frac{\left(1-e^{-\gamma T_{0}}\right)}{\beta \tau}\left[2 \beta^{2} x_{0}^{2}-\left(\mu+\beta x_{0}\right)^{2}\right]\right)<\lambda_{0}<$
$\quad \frac{2 \beta x_{0}^{2}\left(1-e^{-\gamma T_{0}}\right)}{\tau}$.
(H2) $\frac{2 \beta x_{0}^{2}\left(1-e^{-\gamma T_{0}}\right)}{\tau}<\lambda_{0}<\left(\frac{1-\left(1-\left(\mu+\beta x_{0}\right) T_{0}\right)^{2}}{T_{0}^{2}}+2 \beta^{2} x_{0}^{2}\right) \frac{\left(1-e^{-\gamma T_{0}}\right)}{\beta \tau}$.

We have the following result.

Theorem 2.1: If one of the hypothesis $(\mathbf{H 1})$ or $(\mathbf{H 2})$ is satisfied, then there exists $\epsilon_{0}>0$ such that for $0<T_{0}<\epsilon_{0}$ the trivial periodic solution is exponentially stable.

Proof: We have $D_{X} \Psi\left(T_{0}, X\right)=D_{X} \Theta\left(\Phi\left(T_{0}, X\right)\right) \frac{\partial \Phi}{\partial X}\left(T_{0}, X\right)$, then for $X_{0}=(V(0), U(0), P(0))$ we obtain

$$
\begin{aligned}
D_{X} \Psi\left(T_{0}, X\right) & =\left(\begin{array}{lll}
\frac{\partial \Theta_{1}}{\partial V} & \frac{\partial \Theta_{1}}{\partial U} & \frac{\partial \Theta_{1}}{\partial P} \\
\frac{\partial \Theta_{2}}{\partial V} & \frac{\partial \Theta_{2}}{\partial U} & \frac{\partial \Theta_{2}}{\partial P} \\
\frac{\partial \Theta_{3}}{\partial V} & \frac{\partial \Theta_{3}}{\partial U} & \frac{\partial \Theta_{3}}{\partial P}
\end{array}\right)\left(\begin{array}{lll}
\frac{\partial \Phi_{1}}{\partial V} & \frac{\partial \Phi_{1}}{\partial U} & \frac{\partial \Phi_{1}}{\partial P} \\
\frac{\partial \Phi_{2}}{\partial V} & \frac{\partial \Phi_{2}}{\partial U} & \frac{\partial \Phi_{2}}{\partial P} \\
\frac{\partial \Phi_{3}}{\partial V} & \frac{\partial \Phi_{3}}{\partial U} & \frac{\partial \Phi_{3}}{\partial P}
\end{array}\right)\left(T_{0}, X_{0}\right) \\
& =\left(\begin{array}{lll}
\frac{\partial \Phi_{1}}{\partial V} & \frac{\partial \Phi_{1}}{\partial U} & \frac{\partial \Phi_{1}}{\partial P} \\
\frac{\partial \Phi_{2}}{\partial V} & \frac{\partial \Phi_{2}}{\partial U} & \frac{\partial \Phi_{2}}{\partial P} \\
\frac{\partial \Phi_{3}}{\partial V} & \frac{\partial \Phi_{3}}{\partial U} & \frac{\partial \Phi_{3}}{\partial P}
\end{array}\right)\left(T_{0}, X_{0}\right) .
\end{aligned}
$$

From the variational equation we find

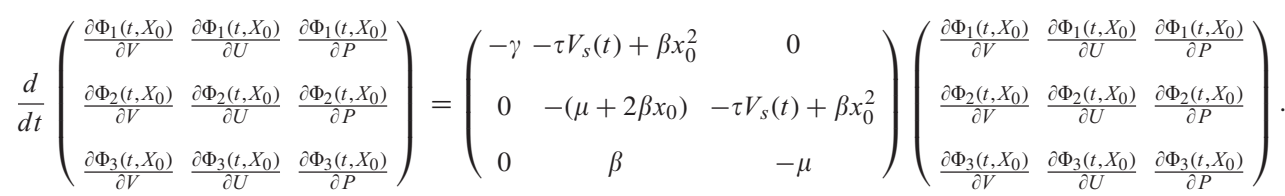

Then we obtain

$$
\begin{gathered}
\frac{\partial \dot{\Phi}_{1}\left(t, X_{0}\right)}{\partial V}=-\gamma \frac{\partial \Phi_{1}\left(t, X_{0}\right)}{\partial V}+\left(-\tau V_{s}(t)+\beta x_{0}^{2}\right) \frac{\partial \Phi_{2}\left(t, X_{0}\right)}{\partial V} \\
\frac{\partial \dot{\Phi}_{2}\left(t, X_{0}\right)}{\partial V}=-\left(\mu+2 \beta x_{0}\right) \frac{\partial \Phi_{2}\left(t, X_{0}\right)}{\partial V}+\left(-\tau V_{s}(t)+\beta x_{0}^{2}\right) \frac{\partial \Phi_{3}\left(t, X_{0}\right)}{\partial V} \\
\text { 01009-p.3 }
\end{gathered}
$$




$$
\begin{gathered}
\frac{\partial \dot{\Phi}_{3}\left(t, X_{0}\right)}{\partial V}=\beta \frac{\partial \Phi_{2}\left(t, X_{0}\right)}{\partial V}-\mu \frac{\partial \Phi_{3}\left(t, X_{0}\right)}{\partial V} \\
\frac{\partial \dot{\Phi}_{1}\left(t, X_{0}\right)}{\partial U}=-\gamma \frac{\partial \Phi_{1}\left(t, X_{0}\right)}{\partial U}+\left(-\tau V_{s}(t)+\beta x_{0}^{2}\right) \frac{\partial \Phi_{2}\left(t, X_{0}\right)}{\partial U} \\
\frac{\partial \dot{\Phi}_{2}\left(t, X_{0}\right)}{\partial U}=-\left(\mu+2 \beta x_{0}\right) \frac{\partial \Phi_{2}\left(t, X_{0}\right)}{\partial U}+\left(-\tau V_{s}(t)+\beta x_{0}^{2}\right) \frac{\partial \Phi_{3}\left(t, X_{0}\right)}{\partial U} \\
\frac{\partial \dot{\Phi}_{3}\left(t, X_{0}\right)}{\partial U}=\beta \frac{\partial \Phi_{2}\left(t, X_{0}\right)}{\partial U}-\mu \frac{\partial \Phi_{3}\left(t, X_{0}\right)}{\partial U} \\
\frac{\partial \dot{\Phi}_{1}\left(t, X_{0}\right)}{\partial P}=-\gamma \frac{\partial \Phi_{1}\left(t, X_{0}\right)}{\partial P}+\left(-\tau V_{s}(t)+\beta x_{0}^{2}\right) \frac{\partial \Phi_{2}\left(t, X_{0}\right)}{\partial P} \\
\frac{\partial \dot{\Phi}_{2}\left(t, X_{0}\right)}{\partial P}=-\left(\mu+2 \beta x_{0}\right) \frac{\partial \Phi_{2}\left(t, X_{0}\right)}{\partial P}+\left(-\tau V_{s}(t)+\beta x_{0}^{2}\right) \frac{\partial \Phi_{3}\left(t, X_{0}\right)}{\partial P} \\
\frac{\partial \dot{\Phi}_{3}\left(t, X_{0}\right)}{\partial P}=\beta \frac{\partial \Phi_{2}\left(t, X_{0}\right)}{\partial P}-\mu \frac{\partial \Phi_{3}\left(t, X_{0}\right)}{\partial P}
\end{gathered}
$$

From (3)-(4) we obtain $\frac{\partial \Phi_{2}\left(t, X_{0}\right)}{\partial V}=0, \frac{\partial \Phi_{3}\left(t, X_{0}\right)}{\partial V}=0$ and $\frac{\partial \Phi_{1}\left(t, X_{0}\right)}{\partial V}=e^{-\gamma t}$.

To solve the system (3)-(10) we use power series. Put

$$
\begin{array}{ll}
\frac{\partial \Phi_{1}\left(T_{0}, X_{0}\right)}{\partial U}=\sum_{n \geq 0} a_{n} T_{0}^{n}, & a_{0}=0, \\
\frac{\partial \Phi_{1}\left(T_{0}, X_{0}\right)}{\partial P}=\sum_{n \geq 0} b_{n} T_{0}^{n}, & b_{0}=0, \\
\frac{\partial \Phi_{2}\left(T_{0}, X_{0}\right)}{\partial U}=\sum_{n \geq 0} c_{n} T_{0}^{n}, & c_{0}=1, \\
\frac{\partial \Phi_{2}\left(T_{0}, X_{0}\right)}{\partial P}=\sum_{n \geq 0} d_{n} T_{0}^{n}, & d_{0}=0, \\
\frac{\partial \Phi_{3}\left(T_{0}, X_{0}\right)}{\partial U}=\sum_{n \geq 0} e_{n} T_{0}^{n}, & e_{0}=0, \\
\frac{\partial \Phi_{3}\left(T_{0}, X_{0}\right)}{\partial P}=\sum_{n \geq 0} f_{n} T_{0}^{n}, & f_{0}=1 .
\end{array}
$$

Hence

$$
\operatorname{det}\left(D_{X} \Psi\left(T_{0}, X\right)-v I\right)=\left(e^{-\gamma T_{0}}-v\right)\left(v^{2}+A v+B\right)
$$


where

$$
\begin{gathered}
A=-\left(\sum_{n \geq 0} c_{n} T_{0}^{n}+\sum_{n \geq 0} a_{n} T_{0}^{n}\right), \\
B=\left(\sum_{n \geq 0} c_{n} T_{0}^{n}\right)\left(\sum_{n \geq 0} a_{n} T_{0}^{n}\right)+\left(\sum_{n \geq 0} d_{n} T_{0}^{n}\right)\left(\sum_{n \geq 0} e_{n} T_{0}^{n}\right) .
\end{gathered}
$$

We have an eigenvalue $\nu_{1}=e^{-\gamma T_{0}}<1$, it remains to find the two other eigenvalues $\nu_{2}$ and $\nu_{3}$.

The discriminant of the polynom $v^{2}+A v+B$ is

$$
\Delta=4 \beta T_{0}^{2}\left(2 \beta x_{0}^{2}-\frac{\tau \lambda_{0}}{1-e^{-\gamma T_{0}}}\right)+o\left(T_{0}^{2}\right)=4 \beta T_{0}^{2} \Delta_{1}+o\left(T_{0}^{2}\right)
$$

where $\Delta_{1}=2 \beta x_{0}^{2}-\frac{\tau \lambda_{0}}{1-e^{-\gamma T_{0}}}$.

For $T_{0}$ small enough, we deduce the sign of $\Delta$ from that of $\Delta_{1}$.

1. $\Delta_{1}>0$ if an only if $\lambda_{0}<\frac{2 \beta x_{0}^{2}\left(1-e^{-\gamma T_{0}}\right)}{\tau}$.

For $T_{0}$ small enough, we have

$$
\nu_{2,3}=1-\left(\mu+\beta x_{0}\right) T_{0} \pm T_{0} \sqrt{2 \beta^{2} x_{0}^{2}-\frac{\beta \tau \lambda_{0}}{1-e^{-\gamma T_{0}}}+o(1)} .
$$

The solution $\xi$ is exponentially stable if

$$
\left|v_{i}\right|<1 \text { for } i=1,2,3 \text {. }
$$

That is

$$
\begin{gathered}
T_{0}<\frac{2}{\mu+\beta x_{0}}, \\
\lambda_{0}>\frac{\left(1-e^{-\gamma T_{0}}\right)}{\beta \tau}\left[2 \beta^{2} x_{0}^{2}-\left(\frac{2}{T_{0}}-\left(\mu+\beta x_{0}\right)\right)^{2}+o(1)\right],
\end{gathered}
$$

and

$$
\lambda_{0}>\frac{\left(1-e^{-\gamma T_{0}}\right)}{\beta \tau}\left[2 \beta^{2} x_{0}^{2}-\left(\mu+\beta x_{0}\right)^{2}+o(1)\right] .
$$

Then we deduce that $\xi$ is exponentially stable if (H1) is satisfied and $\epsilon_{0} \leq \frac{2}{\mu+\beta x_{0}}$.

2. $\Delta_{1}<0$ if an only if $\lambda_{0}>\frac{2 \beta x_{0}^{2}\left(1-e^{-\gamma T_{0}}\right)}{\tau}$.

For $T_{0}$ small enough, we have

$$
v_{2,3}=1-\left(\mu+\beta x_{0}\right) T_{0} \pm i T_{0} \sqrt{-2 \beta^{2} x_{0}^{2}+\frac{\beta \tau \lambda_{0}}{1-e^{-\gamma T_{0}}}+o(1)} .
$$

That is

$$
\lambda_{0}<\left(\frac{1-\left(1-\left(\mu+\beta x_{0}\right) T_{0}\right)^{2}}{T_{0}^{2}}+2 \beta^{2} x_{0}^{2}+o(1)\right) \frac{\left(1-e^{-\gamma T_{0}}\right)}{\beta \tau} .
$$

Then we deduce that $\xi$ is exponentially stable if $(\mathbf{H 2})$ is satisfied and $\epsilon_{0} \leq \frac{2}{\mu+\beta x_{0}}$. 


\section{Analysis of the general model}

In this section we consider the general model (1), we use the evolution semi group theory to prove the existence of mild solutions (see [4]).

We consider the case where $\beta(x) \equiv \beta$ and $\mu(x) \equiv \mu$ are constant. Then for $t \in J, x \in Y:=$ $\left[x_{0},+\infty\right), u^{0} \in D$ and $u \in L^{1}(Y)$, we may rewrite (1.1) as

$$
\left\{\begin{array}{l}
v^{\prime}(t)=-\gamma v(t)-\tau v(t)|u|_{1}+\beta x_{0}^{2}|\kappa u|_{1}, \quad t \neq t_{i}, \\
v\left(t_{i}^{+}\right)-v\left(t_{i}\right)=\lambda_{i}, \lambda_{i}>0, i \in n^{*}, \\
v(0)=v^{0}
\end{array}\right.
$$

and

$$
\left\{\begin{array}{l}
\partial_{t} u(t, x)+\tau v(t) \partial_{x} u(t, x)+(\mu+\beta) u(t, x)=2 \beta \int_{x}^{\infty} \kappa(x, y) u(y) d y, \\
u\left(t, x_{0}\right)=0, u(0, x)=u^{0}(x),
\end{array}\right.
$$

where $|u|_{1}=\int_{x_{0}}^{\infty}|u(y)| d y$.

Set $D:=\left\{u^{0} \in L^{1}(Y) \cap W^{1,1}(\mathbb{R}): x^{2} u^{0},\left(u^{0}\right)^{\prime}, x\left(u^{0}\right)^{\prime} \in L^{1}(Y), u^{0}(x)=0\right.$ for $\left.x \leq x_{0}\right\}$.

Let $J_{i}=\left(t_{i}, t_{i+1}\right), i=0, \ldots, p$, and $v_{i}$ be the restriction of a function $y$ to $J_{i}$.

Consider the following spaces

$P C=\left\{v: J \rightarrow X, v_{i} \in C\left(J_{i}, X\right), i=0, \ldots, p\right.$, such that $v\left(t_{i}^{-}\right)$and $v\left(t_{i}^{+}\right)$exist and satisfy $v\left(t_{k}^{-}\right)=v\left(t_{k}\right)$ for $\left.i=0, \ldots, p\right\}$

with the norm $\|v\|_{P C}=\max \left\{\left\|v_{k}\right\|_{\infty}, \quad i=0, \ldots, p\right\}$,

$P C^{1}(J, \mathbb{R})=\left\{v \in P C: v \in C^{1}\left(J_{i}, \mathbb{R}\right), \exists v^{\prime}\left(t_{i}^{+}\right), v^{\prime}\left(t_{i}^{-}\right), i=1, \ldots, p\right\}$

with the norm $\|v\|_{P C^{1}}=\max \left\{\|v\|_{P C},\left\|v^{\prime}\right\|_{P C}\right\}$ and

$X:=L^{1}(Y ;(a+x) d x)$ where $a>0$, with the norm defined by $\|y\|_{X}=a|y|_{1}+|x y|_{1}$.

Then $\left(P C,\|\cdot\|_{P C}\right),\left(P C^{1},\|\cdot\|_{P C^{1}}\right)$ and $\left(X,\|\cdot\|_{X}\right)$ are Banach spaces.

For $\bar{u} \in C(J, X)$, the solution of (12) is given by

$$
\begin{gathered}
v_{\bar{u}}(t)=\sum_{0<t_{j} \leq t \leq b} \lambda_{j} e^{\left(-\gamma\left(t-t_{j}\right)-\tau \int_{t_{j}}^{t}|\bar{u}(s)|_{1} d s\right)}+v^{0} e^{\left(-\gamma t-\tau \int_{0}^{t}|\bar{u}(s)|_{1} d s\right)} \\
+\beta x_{0}^{2} \int_{0}^{t}|\kappa \bar{u}(s)|_{1} e^{\left(-\gamma(t-s)-\tau \int_{s}^{t}|\bar{u}(\sigma)|_{1} d \sigma\right)} d s
\end{gathered}
$$

Let $v=v_{\bar{u}}$, the problem (13) is written as

$$
\left\{\begin{array}{l}
u^{\prime}(t)+A_{v}(t) u(t)=f(t, u(t)), \quad t \in J, \\
u(0)=u_{0}
\end{array}\right.
$$

where

$$
A_{v_{\bar{u}}}(.)(u(x))=v_{\bar{u}}(.) \partial_{x} u(x)+(\mu+\beta) u(x), \quad \text { for } x \in Y
$$

and

$$
f(t, u(t, x))=2 \beta \int_{x}^{\infty} \kappa(x, y) u(t, y) d y, \quad \text { with } \quad f: J \times X \rightarrow X .
$$

Then for $(s, t) \in \Delta:=\left\{(t, s) \in J^{2}, t \geq s\right\}$, the evolution problem for (13) is given by (see [1], [5])

$$
\left[U_{v_{\bar{u}}}(t, s) u^{0}\right](x)=u^{0}\left(x-\int_{s}^{t} v_{\bar{u}}(\sigma) d \sigma\right) e^{-\phi(t, s)},
$$

where $\phi(t, s)=(\mu+\beta)(t-s)$. 
Remark 3.1: The two parameter family linear operators $U_{v_{\bar{u}}}(t, s)$ is an exponentially bounded evolution semi-group system (see [4]).

For $v=v_{\bar{u}}$ the solution of (13) is given by

$$
u(t)=U_{v_{\bar{u}}}(t, 0) u^{0}+\int_{0}^{t} U_{v_{\bar{u}}}(t, s) f(s, u(s)) d s .
$$

Theorem 3.2: The problem (13) has a unique mild solution $u \in C(J, X)$.

Proof: Let $u_{1}, u_{2} \in X$ then for $t \in J$ and $x \geq x_{0}$, we have

$$
\begin{aligned}
\left\|f\left(t, u_{1}\right)-f\left(t, u_{2}\right)\right\|_{X} & \leq \int_{x_{0}}^{\infty} 2 \beta\left|\int_{x}^{\infty} \kappa(x, y)\left(u_{1}(y)-u_{2}(y)\right) d y\right|(a+x) d x \\
& \leq 2 \beta \int_{x_{0}}^{\infty}\left|u_{1}(y)-u_{2}(y)\right| d y \int_{x_{0}}^{y} \kappa(x, y)(a+x) d x \\
& \leq 2 \beta \int_{x_{0}}^{\infty}\left|u_{1}(y)-u_{2}(y)\right| \frac{\left(a y+\frac{y^{2}}{2}\right)}{y} d y \\
& \leq 2 \beta \int_{x_{0}}^{\infty}\left|u_{1}(y)-u_{2}(y)\right|(a+y) d y \\
& \leq 2 \beta\left\|u_{1}-u_{2}\right\|_{X} .
\end{aligned}
$$

Then we deduce that (13) has a unique mild solution in $X$ given by (16).

Theorem 3.3: For $v^{0}>0$ and $u^{0} \in X$, the problem (12) and (13) has a unique global positive solution $(v, u) \in P C^{1}(J, \mathbf{R}) \times C(J, X)$.

Proof: Let $\bar{u}_{1}, \bar{u}_{2} \in C(J, X)$, from the explicit representation of $v_{\bar{u}}$ we have

$\left|v_{\bar{u}_{1}}(t)-v_{\bar{u}_{2}}(t)\right| \leq \leq b \tau\left(\sum_{0 \leq t_{j} \leq t} \lambda_{j}+v^{0}+\beta x_{0}\right)\left\|\bar{u}_{1}-\bar{u}_{2}\right\|_{\infty}$.

Let $\Lambda(\bar{u})(t)=U_{v_{\bar{u}}}(t, 0) u^{0}$, for $t \in J$ and $\bar{u} \in C(J, X)$.

Next we show that $\Lambda: C(J, X) \rightarrow C(J, X)$ is a contraction, which would imply existence and uniqueness of the solution of (12) and (13) (see [2]).

In fact, for $\bar{u}_{1}, \bar{u}_{2} \in C(J, X)$ and $t \in J$, we have

$\left\|\Lambda\left(\bar{u}_{1}\right)(t)-\Lambda\left(\bar{u}_{2}\right)(t)\right\|_{X} \leq\left|u^{0}\right|_{1} b^{2} \tau\left(\sum_{0 \leq t_{j} \leq t} \lambda_{j}+v^{0}+\beta x_{0}\right)\left\|\bar{u}_{1}-\bar{u}_{2}\right\|_{\infty}$.

Hence $\Lambda$ is a contraction for $\left|u^{0}\right|_{1}\left(\sum_{0 \leq t_{j} \leq b} \lambda_{j}+v^{0}+\beta x_{0}\right)<\frac{1}{b^{2} \tau}$.

Now, we prove the existence and uniqueness of solution for (12) and (13). Let $r>0$ such that $u^{0} \in B(0, r) \subset X$, then there exists $K>0$ such that

$$
\left\|U_{v_{u}}(t, s)\right\| \leq K \text { for all } u \in B(0, r) .
$$

Let $u \in C(J, X)$ such that $u(t)=U_{v_{u}}(t, 0) u^{0}+\int_{0}^{t} U_{v_{u}}(t, s) f(s, u(s)) d s, \quad t \in J$ and $u(t) \in B(0, r)$, $t \in J$, then

$$
\|u(t)\|_{X} \leq\left|u^{0}\right|_{1} K+2 \beta K b r .
$$

Assume that $\left|u^{0}\right|_{1} K+2 \beta K b r \leq r$ and set $C=\left\{u \in C(J, X):\|u\|_{\infty} \leq r\right\}$. 
Now, we show that $N: C \rightarrow C$ has a unique fixed point.

Let $u_{1}, u_{2} \in C$, thus

$$
\left\|\left(N u_{1}\right)(t)-\left(N u_{2}\right)(t)\right\|_{X} \leq e^{\mu t}\left(c+\frac{2 \beta\left(K x_{0}+r\right)}{x_{0} \mu}\right)\left\|u_{1}-u_{2}\right\|_{*}
$$

where $\|u\|_{*}=\sup _{t \in J} e^{-\mu t}\|u(t)\|_{X}, c=\left|u^{0}\right|_{1} b^{2} \tau\left(\sum_{0 \leq t_{j} \leq t} \lambda_{j}+v^{0}+\beta x_{0}\right)$ and $\mu>0$ large enough such that $C_{0}=c+\frac{2 \beta\left(K x_{0}+r\right)}{x_{0} \mu}<1$.

Hence $\left\|N u_{1}-N u_{2}\right\|_{*} \leq C_{0}\left\|u_{1}-u_{2}\right\|_{*}$.

To prove the positivity of the solution of (13), we proceed by induction.

For $n \geq 1$ we put

$$
u_{n+1}(t)=u_{1}(t)+\int_{s}^{t} U_{v}(t, s) f\left(s, u_{n}(s)\right) d s, t \geq 0 .
$$

And we conclude that the solution for the problem (13) is positive.

\section{Remarks}

In this work we have studied a prion disease model with impulse effects. First we have transformed the general model into impulsive differential equation system, which help to study the exponential stability of the trivial periodic equilibrium. After that, we have studied the general problem, we have proved the existence of solutions under conditions on the parameters of the model. It will be very interesting to search a global solutions on $\mathbb{R}_{+}$in order to study their stabilities and to give numerical simulations to illustrate the results obtained.

\section{References}

[1] K.J. Engel and R. Nagel, One-Parameter Semigroups for Linear Evolution Equations, Graduate Texts in Mathematics, Springer-Verlag, New York 194 (2000).

[2] A. Granas and J. Dugundji, Fixed Point Theory, Springer-Verlag, New York, 2003.

[3] M. Greer, A population model of prion dynamics, Ph.D. Thesis, Vanderbilt University, Nashville (2002).

[4] A. Lakmeche, I. Mammar and A. Ouahab, Existence Results for Prion Disease Model with Impulse Effects, Communications on Applied Nonlinear Analysis, 21 (2014) 3, 41-52.

[5] A. Pazy, Semigroups of Linear Operators and Applications to Partial Differential Equations, Springer-Verlag, New York, 1983.

[6] J. Pruss, L. Pujo-Menjouet, G. Webb and R. Zacher, Analysis of a model for the dynamics of prions, Discrete and continous dynamical systems, 6 (2006) 225-235. 